\title{
Establishment of a dual-color fluorescence tracing orthotopic transplantation model of hepatocellular carcinoma
}

\author{
QIAN CHEN ${ }^{1 *}$, XIAOPING WANG ${ }^{1 *}$, HAO WU $^{1}$, HUI WANG $^{1}$, MINGAO ZHU $^{1}$, ROUSHU WANG $^{1}$, YING WU $^{1}$, \\ LUYAO ZHANG $^{1}$, QIAO MENG ${ }^{1}$, RANRAN SONG ${ }^{1}$, ZHIXIANG ZHUANG ${ }^{1}$ and QIANG HUANG ${ }^{2}$ \\ Departments of ${ }^{1}$ Oncology and ${ }^{2}$ Neurosurgery, The Second Affiliated Hospital of Soochow University, \\ Suzhou, Jiangsu 215004, P.R. China
}

Received February 11, 2015; Accepted October 26, 2015

DOI: $10.3892 / \mathrm{mmr} .2015 .4624$

\begin{abstract}
Different experimental models of hepatocellular carcinoma (HCC) have been used to investigate the biological mechanisms of hepatocarcinogenesis and its progression. However, previous studies have highlighted the difficulty of distinguishing between the tumor cells and stroma in experimental models of HCC. Therefore the aim of the present study was to establish a red-green dual-color fluorescence tracing orthotopic transplantation model of HCC, and investigate its practical values. Stable high red fluorescent protein (RFP)-expressing HepG2 human hepatoma cells and Hepa1-6 mice hepatoma cells were injected into the right liver lobe of green fluorescent protein-expressing nude mice. The growth and metastasis of the tumors were visualized using a whole-body in vivo fluorescence imaging system in real time. HCC tissues were extracted from tumor-bearing mice, and cut into $5-\mu \mathrm{m}$ serial frozen slices. The organizational structure of the transplanted tumors was observed under a microscope. A dual-color fluorescence tracing orthotopic transplantation tumor model of HCC was successfully established with a success rate of $100 \%$. The growth and metastasis of the tumors were visualized at each stage of development in the tumor-bearing mice. Tumor cells with red fluorescence and host cells with green fluorescence were identified to merge in the reconstruction region of tumor tissue. The invasion, migration, and cell fusion between tumor and host cells was observed clearly. The dual-color fluorescence tracing orthotopic transplantation model of HCC was determined to be a stable and reliable method for tracking tumor progression.
\end{abstract}

Correspondence to: Professor Zhixiang Zhuang, Department of Oncology, The Second Affiliated Hospital of Soochow University, 1055 Sanxiang Road, Suzhou, Jiangsu 215004, P.R. China

E-mail: sdfeyzzx@sina.com

"Contributed equally

Key words: hepatocellular carcinoma, orthotopic transplantation mode, dual-color fluorescence imaging, tumor invasion, cell fusion
Mutual interactions between hepatoma cells and host tissues may be observed directly using this model, further elucidating the development of the tumor microenvironment.

\section{Introduction}

Primary liver cancer is the fifth most common type of cancer worldwide and the third most common cause of cancer-related mortality $(1,2)$. Hepatocellular carcinoma (HCC) accounts for $85-90 \%$ of primary liver cancers (2). Progress has been made in detecting and treating localized disease, however the 5-year survival of patients with liver cancer was $\sim 15 \%$ in the USA in $2002-2008, \sim 12 \%$ in Europe in 2000-2007, and 5\% in low-income countries in 2002 (3). Thus, establishing an appropriate animal model is critical for understanding the molecular, cellular and pathophysiological mechanisms of HCC, and is essential for the development of novel therapeutic strategies.

The constant evolution of model design and technological development allows for numerous experimental models of HCC to be developed including spontaneous, induced, transplantable, and genetically engineered models (4). Currently, the most commonly employed models of HCC are transplantable ones including subcutaneous and orthotopic transplantation in nude mice (4). However, they present a difficulty in distinguishing the difference in position and morphology between the tumor cells and the stroma. The lack of information regarding the interaction between tumor and host is largely due to the absence of suitable models that allow visualization and precise investigation of the tumor-host interaction in vivo (5). The introduction of fluorescent protein visualization to this field allows for the labeling of host and tumor cells with different color fluorescence proteins, thus enabling investigation of the tumor microenvironment (TME). Previous studies have reported a dual-color fluorescence tracing transplantation model in various tumor types (5-9). However, to the best of our knowledge, a dual-color fluorescence tracing orthotopic transplantation model of HCC has not been established. Therefore, the current study aimed to establish a dual-color fluorescence tracing orthotopic transplantation model of HCC, based on green fluorescence protein (GFP)-expressing nude mice and red fluorescence protein (RFP)-expressing hepatoma cells. 


\section{Materials and methods}

Cell culture. The HepG2 human hepatoma cell line and Hepa1-6 mice hepatoma cell line (Type Culture Collection of the Chinese Academy of Sciences, Shanghai, China) were cultured in Dulbecco's modified Eagle's medium (HyClone; GE Healthcare Life Sciences, Beijing, China) supplemented with $10 \%$ fetal bovine serum (Gibco; Thermo Fisher Scientific, Inc., Waltham, MA, USA) in a humidified atmosphere of $5 \% \mathrm{CO}_{2}$ at $37^{\circ} \mathrm{C}$.

Red fluorescence labeling of HCC cell lines. According to the manufacturer's instructions, HepG2 and Hepa1-6 HCC cell lines were transfected with RFP gene using a lentivirus-mediated gene transfection kit (pLenO-RIP; Shanghai Innovation Biotechnology Co., Ltd., Shanghai, China). HepG2 and Hepa1-6 cells were then respectively cultured in growth medium to $30-50 \%$ confluence at the time of transduction ( $1 \times 10^{5}$ cells/well in 24 -well plates). Then the cells were incubated with the RFP-lentivirus at a multiplicity of infection of 10 for HepG2 cells and 5 for Hepa1- 6 cells. After $72 \mathrm{~h}$ the positive transduction rate was visualized using fluorescence microscopy. The cells were then passaged at a ratio of $1: 3$ in a selective medium that contained $10 \mu \mathrm{g} / \mathrm{ml}$ puromycin (Sigma-Aldrich, St Louis, MO, USA). Cell clones with high RFP-expression were selected in 96-well plates. They were amplified and transferred by conventional culture methods. The cells that underwent successful transduction and screening were termed Hepa1-6-RFP and HepG2-RFP, respectively. Adherent cells were digested in cell suspension and were subsequently assessed by flow cytometry (Beckman Coulter, Miami, FL, USA) to analyze the RFP-positive cells

Animals. NC-C57BL6J-GFP nude mice (Department of Neurosurgery, Second Affiliated Hospital of Suzhou University, Suzhou, China) (10) were housed in microisolator cages with sterile bedding (NASA 1000), water and food was provided ad libitum. The mice were maintained in an environment of $24-26^{\circ} \mathrm{C}$, in a humidity of $50-60 \%$ with $12 \mathrm{~h}$ light/dark cycles. As previously described (10), GFP-expressing nude mice were obtained by crossing non-transgenic $\mathrm{NC}$ athymic nude mice with the GFP transgenic C57BL6J mice. The present study was performed in strict accordance to the recommendations of the Guide for the Care and Use of Laboratory Animals of the National Institutes of Health (publication no. 85-23, revised 1985). The animal use protocol was reviewed and approved by the Institutional Animal Care and Use Committee of Soochow University (Suzhou, China).

Establishment of the dual-color orthotopic transplantation tumor model. A total of 20 NC-C57BL6J-GFP nude mice (age, 6 weeks; body weight, $20 \mathrm{~g}$ ) were inoculated with $1 \times 10^{6} \mathrm{HCC}$ cells. They were divided into two groups: Group I $(n=10)$ were injected with HepG2-RFP cells and group II $(n=10)$ were injected with Hepa1-6-RFP cells. All the surgical procedures were performed under general anesthesia using intraperitoneal injection of $10 \%$ chloral hydrate $(200 \mathrm{mg} / \mathrm{kg}$; Sigma-Aldrich). Once anesthetized, the mice were fixed on an experimental board in a supine position. A 2-mm transverse incision was made below the xiphoid, following sterilization of the area with $70 \%$ alcohol, which was perpendicular to the median line and was $1-1.5 \mathrm{~cm}$ long. The right liver lobes were carefully pulled out of the abdominal cavity with a sterile cotton swab. Tumor cells were resuspended in phosphate-buffered saline. The red fluorescent cell suspension (50 $\mu 1 ; 1 \times 10^{6}$ cells) was injected into the left liver lobes at a depth of $3.5 \mathrm{~mm}$ over $15 \mathrm{~min}$ using a $50 \mu \mathrm{l}$ Hamilton syringe (Anhui Zhenghua Biological Instrument Equipment Co., Ltd., Huaibei, China). Following the injection, a small piece of sterile gauze was placed on the injection site, and light pressure was applied for $1 \mathrm{~min}$ to prevent bleeding and spilling. The skin was then sterilized with 70\% alcohol and the wound sutured with a Plus 5-0 suture line. The mean duration of surgery was $30 \mathrm{~min}$. Postsurgery, mice were treated with $0.1 \mathrm{mg}$ ketoprofen (Sigma-Aldrich) for pain control and were observed continuously for signs of pain or distress (hypoactivity, restlessness, vocalization, hiding, lack of grooming, abnormal posture, tremor or respiratory distress) until they recovered from anesthesia and for the next $48 \mathrm{~h}$. The living conditions of the mice were inspected daily.

Whole-body fluorescence imaging. Following the implantation of tumor cells, the mice were anesthetized via intraperitoneal injection of $10 \%$ chloral hydrate $(200 \mathrm{mg} / \mathrm{kg})$, on week 3,5 and 7 of the current study in order to perform whole-body fluorescent imaging using the in vivo fluorescence imaging system (Kodak, Rochester, NY, USA). The GFP excitation and emission wavelengths were 470 and $535 \mathrm{~nm}$, respectively. The RFP excitation and emission wavelengths were 553 and $574 \mathrm{~nm}$, respectively. Once imaging was complete, each animal was removed from the imaging stage, placed on a heated platform in its original cage and allowed to recover. Subsequent to full recovery from the anesthesia, the animals were returned to the IVC isolation device.

Histological evaluation and subculturing. When tumor-bearing mice appeared distressed (as determined by cachexia, loss of appetite, hypoactivity, lack of grooming or abnormal posture), they were sacrificed by cervical dislocation and an autopsy was conducted. A heart perfusion was performed with 5-10 $\mathrm{ml}$ of 4\% paraformaldehyde. The whole liver was harvested, frozen and sectioned at a thickness of $5 \mu \mathrm{m}$ using conventional culture methods. Samples were then stained with hematoxylin and eosin (Beyotime Institute of Biotechnology, Shanghai, China) using routine histopathological procedures or observed under a fluorescent microscope (Carl Zeiss, Oberkochen, Germany). Cell nuclei were stained blue using 4,6-diamidino-2-phenylindole (Nanjing KeyGen Biotech Co., Ltd, Nanjing, China).

Ascites were obtained from the tumor-bearing mice, were centrifuged and the sediment was cultured in culture medium, containing 10\% FBS. The tumor nodules was harvested and washed with phosphate-buffered saline, containing penicillin and streptomycin, three times. The tumor tissue was subsequently minced with fine scissors into small fragments, and cultured in culture medium, containing 10\% FBS. The medium was replaced with fresh culture medium on the third day. Normal cell culture conditions were maintained for the subsequent days.

\section{Results}

Red fluorescence labeling of HCC cell lines. Lentivirus-mediated RFP gene transfection of HepG2 

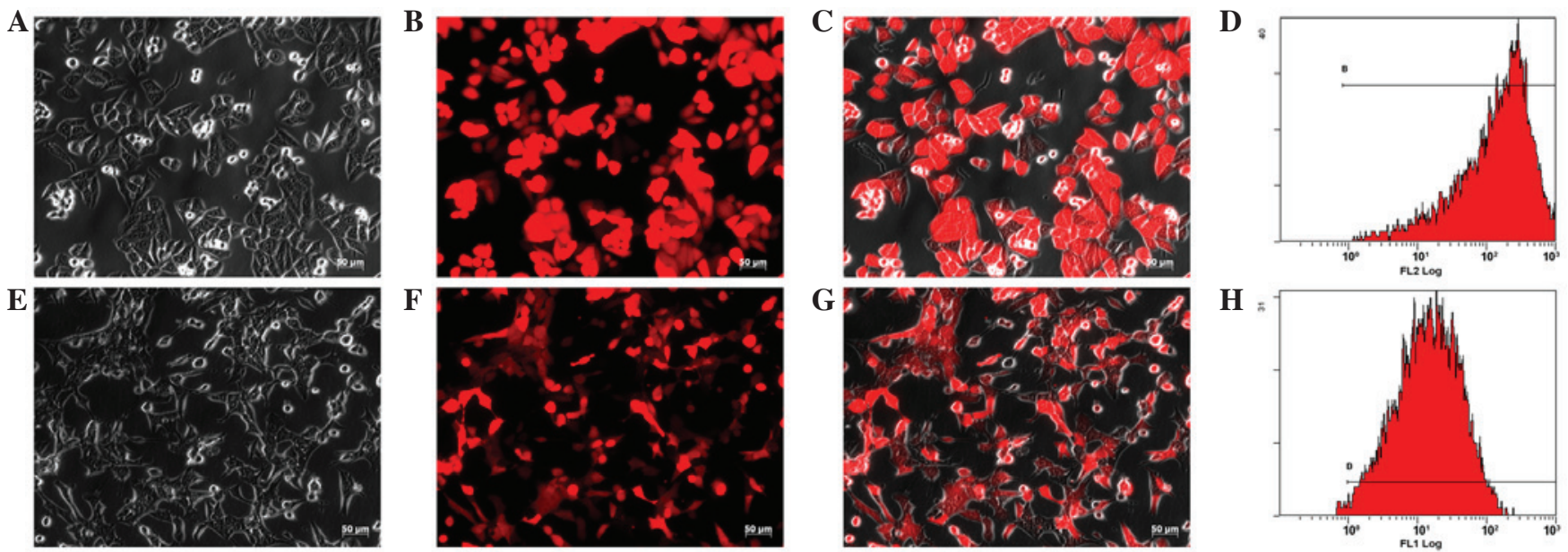

Figure 1. Red fluorescence labeling of hepatocellular carcinoma cell lines. RFP-transfected HepG2 human hepatoma cells were observed under (A) a light microscope or (B) a fluorescence microscope, and (C) the images were merged (scale bar, $50 \mu \mathrm{m}$ ). (D) Flow cytometric analysis demonstrated that $>99 \%$ of the cells expressed RFP. The RFP-transfected Hepa1-6 mouse hepatoma cell line was observed under (E) a light microscope, (F) a fluorescence microscope, and $(\mathrm{G})$ the images were merged (scale bar, $50 \mu \mathrm{m}$ ). (H) Flow cytometric analysis demonstrated that $>98 \%$ of the cells expressed RFP. RFP, red fluorescence protein.

A

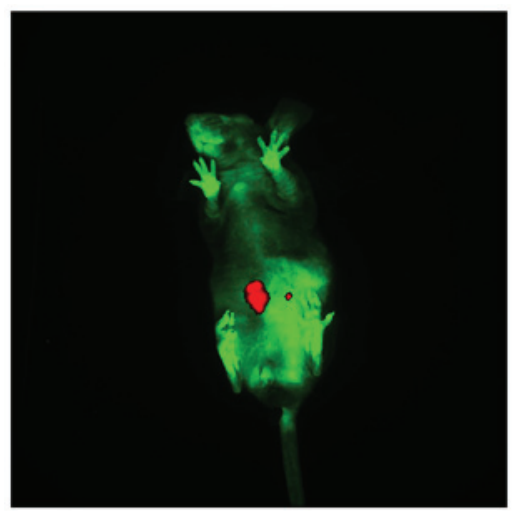

C

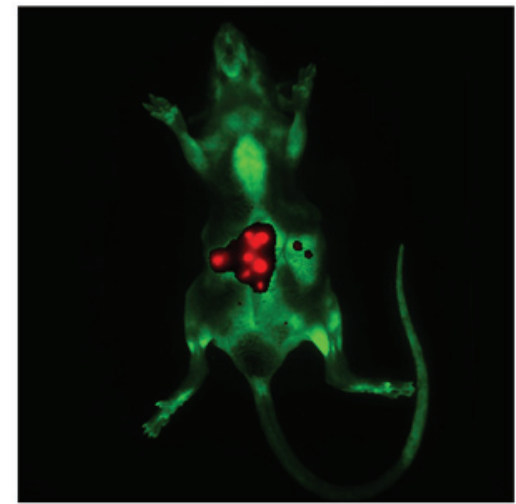

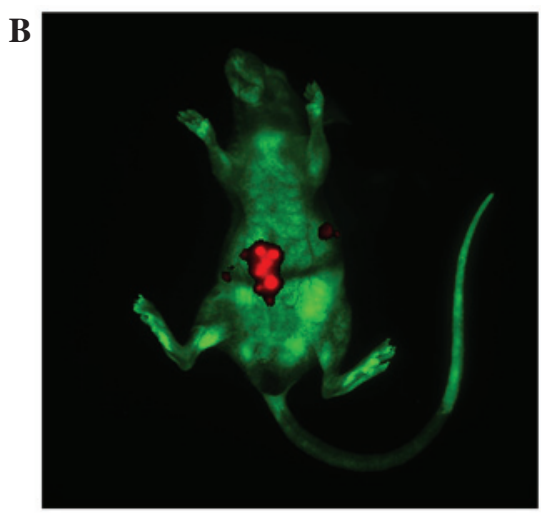

$\mathbf{D}$

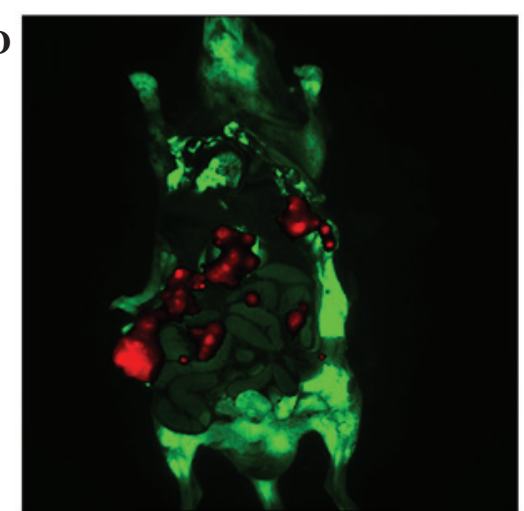

Figure 2. Dual-fluorescence imaging in vivo in tumor-bearing transgenic GFP-nude mice. A HepG2-RFP cell tumor-bearing mouse on week (A) 3, (B) 5 and (C) 7 following implantation of tumor cells. The fluorescence signal intensity indicated xenograft tumor size. (D) The fluorescent imaging following the autopsy of the tumor-bearing mouse. RFP, red fluorescence protein.

and Hepa1-6 cells achieved excellent results as $\sim 100 \%$ of tumor cells expressed RFP under a fluorescence microscope (Fig. 1). Due to the stable integration of the RFP gene in the target cell genome, the expression of RFP in the transfected tumor cells was maintained. Flow cytometric analysis identified that $>98 \%$ of the cells expressed RFP (Fig. 1). The cells exhibited no change in morphology and proliferation following transfection. The HepG2-RFP and Hepa1-6-RFP cells were maintained for $>1$ year, and their expression of the RFP gene remained stable.

Dual-fluorescence imaging in vivo tumor-bearing mice. All mice were alive following the cell transplantation. Fig. 2 demonstrated the dual-fluorescence imaging in the living tumor-bearing mice. RFP signals were used to identify the growth of transplanted tumors in the mice. The intensity of the 
Table I. Oncobiological characteristics of tumor-bearing mice.

\begin{tabular}{lcc}
\hline Oncobiological characteristic & HepG2-RFP cell line & Hepa1-6-RFP cell line \\
\hline Median duration of survival (weeks) & 9 & 5 \\
Orthotopic tumorigenesis & $100 \%(10 / 10)$ & $100 \%(10 / 10)$ \\
Intrahepatic metastases & $100 \%(10 / 10)$ & $100 \%(10 / 10)$ \\
Pulmonary metastases & $0 \%(0 / 10)$ & $20 \%(2 / 10)$ \\
Abdominal wall invasion & $70 \%(7 / 10)$ & $80 \%(8 / 10)$ \\
Peritoneal seeding & $80 \%(8 / 10)$ & $90 \%(9 / 10)$ \\
Bloody ascites & $70 \%(7 / 10)$ & $90 \%(9 / 10)$ \\
Brain metastases & $10 \%(1 / 10)$ & $0 \%(0 / 10)$
\end{tabular}

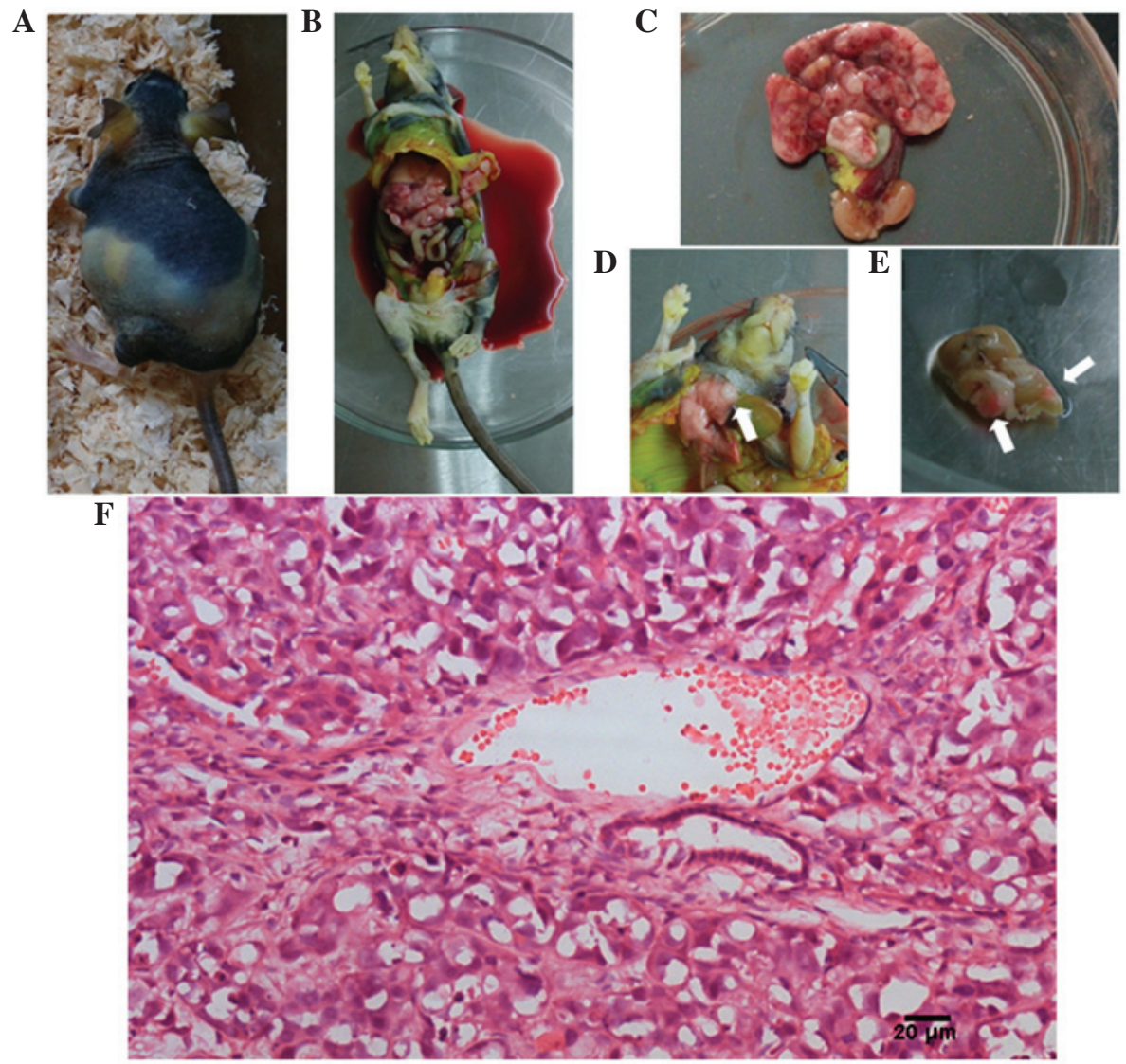

Figure 3. Autopsy and histological evaluation of a tumor-bearing mouse. (A) Increased abdominal girth of mice. Following the autopsy, (B) bloody ascites and abdominal wall invasion, and (C) abdominal cavity metastases and intrahepatic metastases were observed. (D) Lung metastases (arrows). (E) Brain metastases (arrows). (F) Liver tissues from nude mice were detected using hematoxylin and eosin staining (scale bar, $20 \mu \mathrm{m}$ ).

fluorescence signal was associated with the size of the tumor. The signal intensity steadily increased from week 3 to week 7 .

Oncobiological characteristics of nude mice. When the tumor-bearing mice appeared distressed, they were sacrificed and underwent autopsy. The results are displayed in Table I and Fig. 3. The median duration of survival of HepG2-RFP tumor-bearing mice and Hepa1-6-RFP tumor-bearing mice were 9 and 5 weeks, respectively. The rates of spontaneous metastasis of HepG2-RFP tumor-bearing mice and Hepa1-6-RFP tumor-bearing mice reached $100 \%$ each in the liver, 0 and $20 \%$ in the lung, 70 and $80 \%$ in the abdominal wall, 80 and $90 \%$ in the peritoneum, and 10 and 0 in the brain, respectively. A total of 70 and $90 \%$ of HepG2-RFP tumor-bearing mice and Hepa1-6-RFP tumor-bearing mice, respectively, exhibited bloody ascites.

Interactions between tumor cells and host cells. In non-fluorescent tracing solid tumor models, it is often difficult to identify the origin of tumor stroma, and to distinguish between the tumor cells and the stroma. In this dual-color tumor model, transplanted RFP-HCC cells and their descendant cells inside the tumor parenchyma were clearly distinguished from the green host tissue. Mergence was defined as interactions 


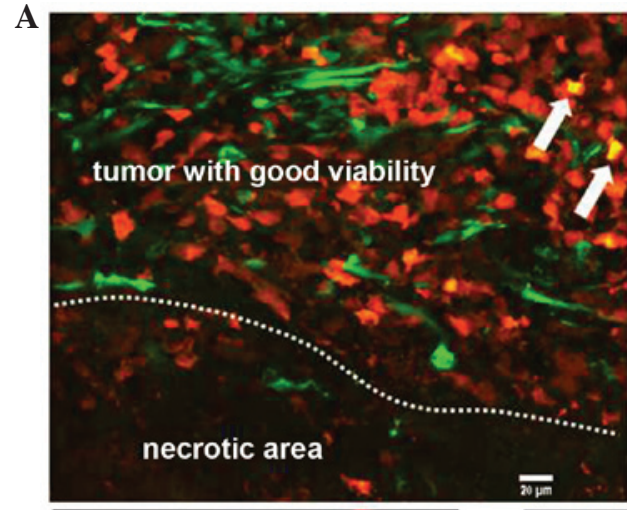

$\mathbf{F}$

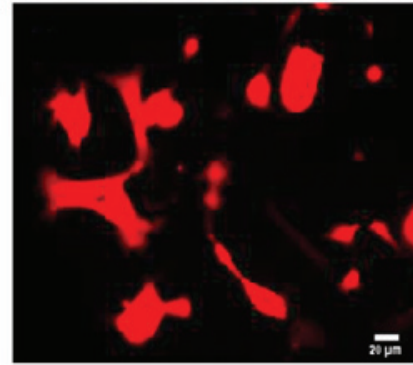

B

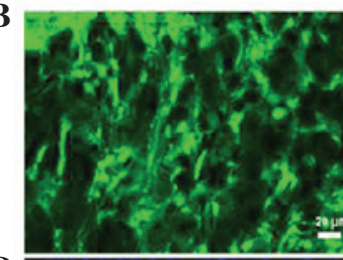

D

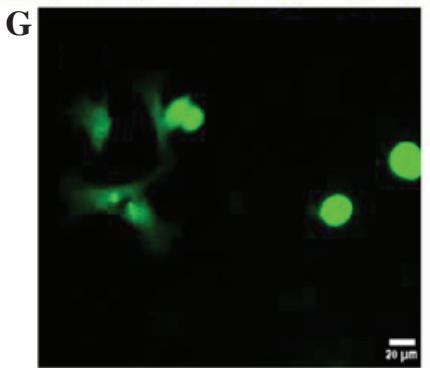

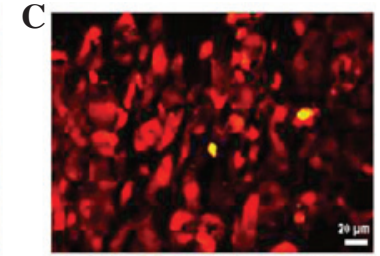

$\mathbf{E}$
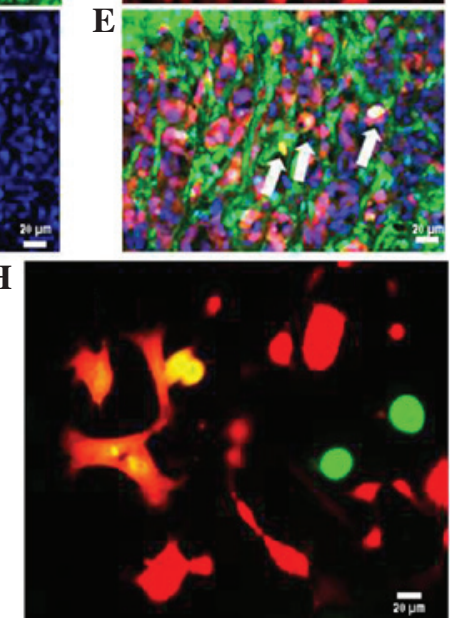

Figure 4. Interweaving distribution and fusion between the tumor cells and host cells (scale bar, $20 \mu \mathrm{m}$ ). In tumor parenchyma, GFP-expressing host cells were observed. (A) RFP-expressing tumor tissue was readily identified in the area where the tumor tissue maintains good viability; however, only the remnants of GFP-expressing tissue can be visualized in the necrotic area. (B) Frozen sections were observed under green fluorescence microscope, (C) red fluorescence microscope, (D) blue fluorescence microscope. (E) In the merged image, orange cells (product of the merging of red and green cells) were identified (arrows) in the tumor parenchyma. (F) In the ascites and tumor nodules of cultured cells, the left six fused cells were observed under red fluorescence microscope, (G) green fluorescence microscope, and $(\mathrm{H})$ merged image. GFP, green fluorescence protein.

between tumor cells and host stroma during tumorigenesis (Fig. 4A-E). The overlapping distribution of tumor cells and host cells was the elemental form of tumor tissue remodeling. In this dual-color xenograft tumor model, apart from RFP and GFP cells, small numbers of orange cells (merging of red and green cells) were also identified (Fig 4A and E). They were considered as hybrids of tumor cells and host cells. To verify the presence of fused cells, ascites and tumor nodules from tumor-bearing mice were cultured according to the method of a previous study (11). These results also identified fused cells in the ascites and tumor nodules of cultured cells (Fig 4F-H).

Tumor cell invasion and migration at the single-cell level. The metastases spawned by carcinomas are formed following the completion of a complex succession of cell biological events collectively termed the invasion-metastasis cascade (12). The exit of tumor cells from their primary sites of growth is the first step in metastatic progression. In the present study, using the frozen tissue sections, the invasion and migration of the tumor cells at the single-cell level was be observed (Fig. 5). It is clear in Fig. 5D that three fluorescent cells have exited the primary tumor site. The initial step of metastasis is the exit of single cells from the primary site.

\section{Discussion}

A well-defined liver cancer model mimicking human liver cancer is essential to reliably reflect the progression of human disease, for basic studies on tumor biology and experimental therapeutic purposes (4). The aim of these models is to provide suitable conditions for the viability of the tumor samples so that they may grow and establish an interaction with the host that resembles the situation observed in the donor. The most commonly employed models for liver cancer are xenograft models including subcutaneous and orthotopic transplant, each having advantages and limitations (4). Subcutaneous xenograft models provide an easy and simple means to implant tumor cells or tissues into the study animal and to monitor tumor size. However, tumor progression in humans is a complicated process in which the interaction of neoplastic cells and the surrounding tumor environment is important (13). A major disadvantage of subcutaneous transplant models is the lack of interaction between the host and the tumor, thus rendering them unable to mimic the TME. By contrast, orthotopic animal models provide highly valuable clinical information including the rate of tumor growth, the therapeutic effect of tested materials, and the in vivo tumor cell behavior as the tumor is located within the targeted organ (14). Thus, orthotopic animal models of HCC have many advantages, including providing highly valuable clinical information on the rate of tumor growth, therapeutic effect of certain materials and the ability to assess the behaviors of tumors in vivo. Differences among these orthotopic animal models were due to the type of the transplantation sample (tumor cell suspension in culture or fresh tumor pieces from surgery). The tumor cell injection animal model is more relevant for clinical practice. However, one drawback of the tumor cell injection model is that it is hard to control tumor cell leakage during injection. In the present study, the cancer cells were directly inoculated into the liver parenchyma. Despite the measures taken to avoid leakage of cancer cells, the possibility of leakage into the 


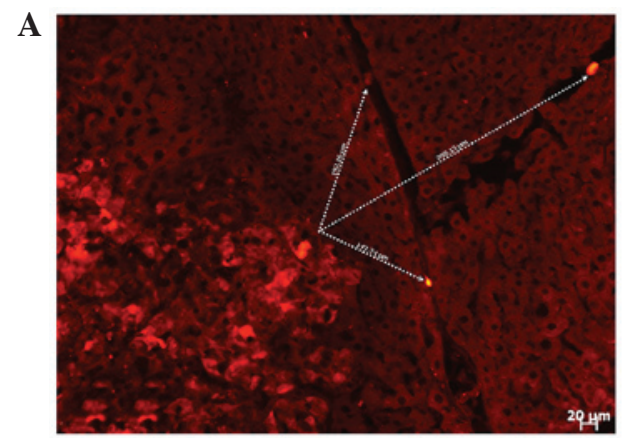

B
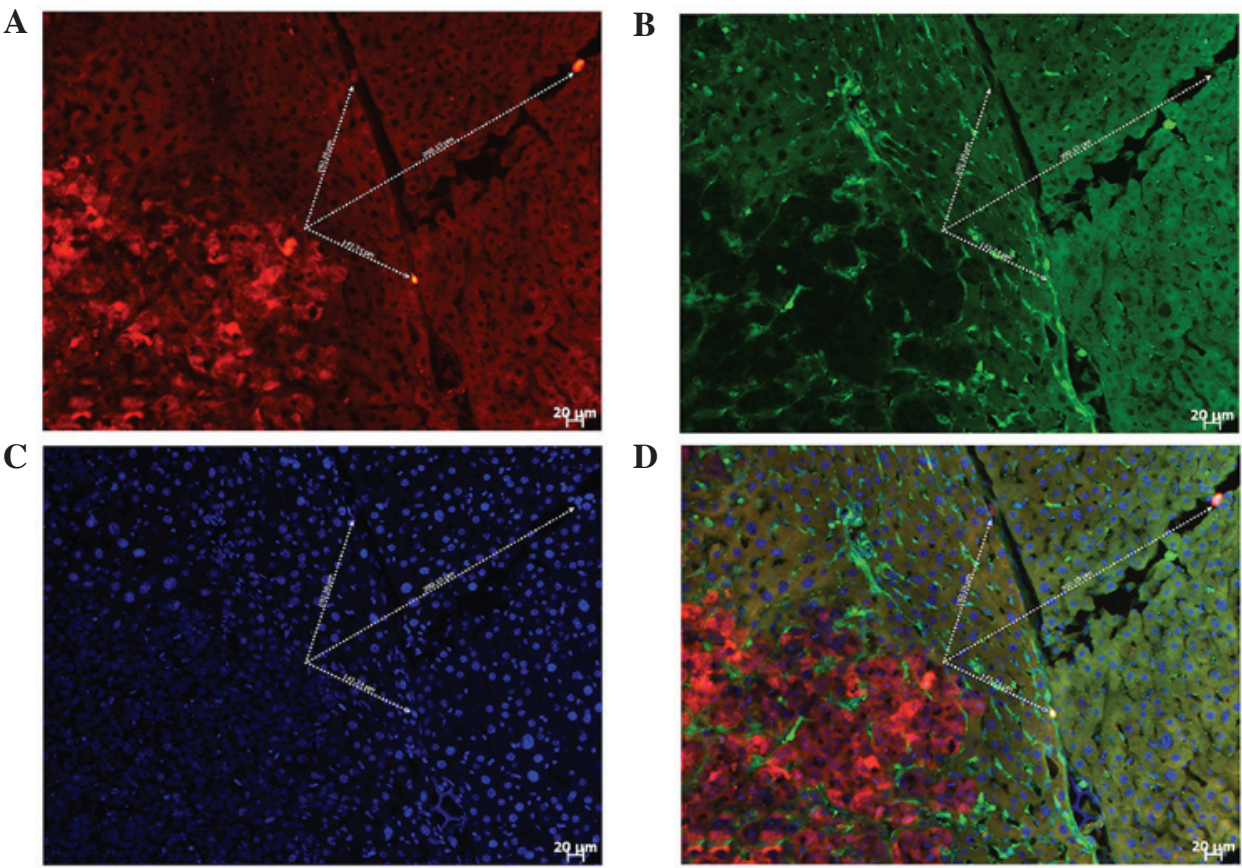

D

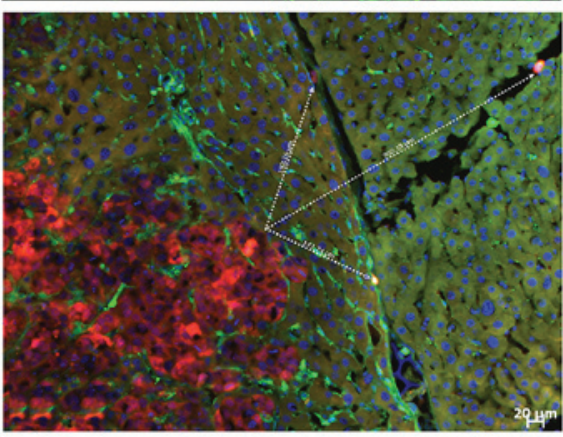

$\mathbf{E}$

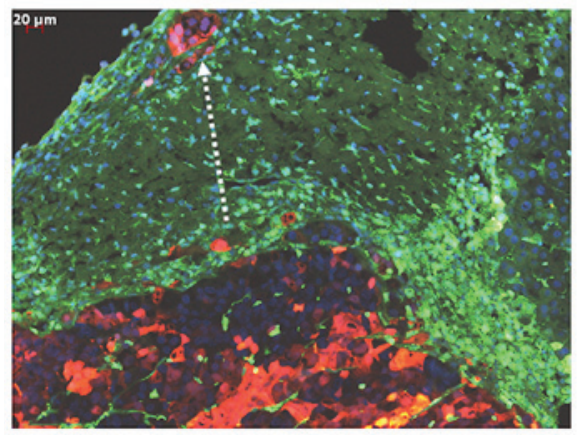

F

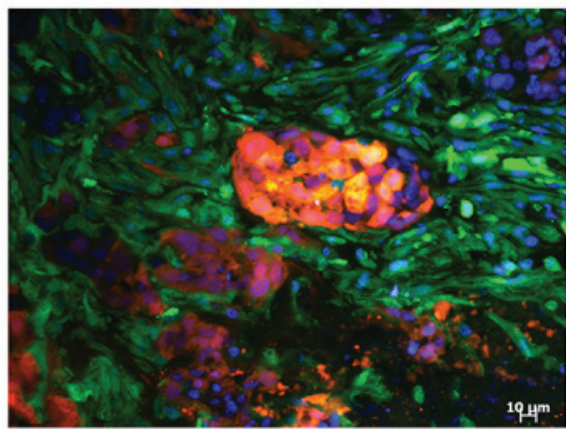

Figure 5. Tumor cell invasion at the single-cell level (scale bar, $20 \mu \mathrm{m}$ ). (A) Frozen sections were observed under red fluorescence, (B) green fluorescence and (C) blue fluorescence. (D) In the merged picture, it is evident that three tumor cells exit their primary sites of growth. The distance travelled by these three tumor cells from their primary sites was 192.16, 392.15 and $147.74 \mu \mathrm{m}$, respectively. (E and F) These migrated tumor cells proliferate and further form distant metastases.

abdominal cavity was not completely eliminated. Therefore, analyses were complicated by the fact that apparent metastasis particularly peritoneal seeding may be due to leakiness. Nevertheless, pulmonary and brain metastases observed in the current study were certainly due to spontaneous metastasis in vivo.

Postoperative evaluation of orthotopic tumors is also a challenge. The most commonly used imaging modalities for orthotopic tumors are ultrasound, computed tomography (CT), magnetic resonance imaging (MRI), and 18F-fluorodeoxyglucose-positron emission tomography (PET)/CT. However, there are drawbacks associated with each method. Ultrasonic examination is inexpensive, however analysis of the data requires a skilled operator (15). $\mathrm{CT} / \mathrm{MRI} / \mathrm{PET}-\mathrm{CT}$ scanning is noninvasive, however the cost is high and it is difficult to perform (16). The use of fluorescent proteins for imaging is revolutionizing in vivo biology. With the use of an in vivo fluorescence imaging system, the transplanted subcutaneous tumors may be measured non-invasively and tumor growth continuously monitored in real time. As a result of the deep position of orthotopic transplantation HCC, it is difficult to identify and measure this at an early stage. Fluorescence technology was introduced for this reason and according to the fluorescent signal intensity, tumor size can be compared.

The progression of a tumor is a multistep process. The TME is critical for malignancy, which is in part the product of the interaction between different cancer types and their host cells. The development of solid tumors is due to the remodeling processes of tumor cells against host tissue. However, the mechanism of this remodeling process and the relevant dynamic changes remain unknown. The dual-fluorescent xenograft HCC model offers a platform to directly monitor multiple interactions between RFP-labeled human tumor cells and GFP-labeled murine host cells. It was determined that dynamic interweaving always exists in the remodeling process of tumor and host tissues. Well grown tumor sites are normally located at those sites where tumor cells and host cells are interweaving distribution. However, in the necrotic area, the host cells have a small proportion. Overall, the TME is provided by the host, however, it is also transformed constantly by tumor cells.

Cell fusion is not only a common physiological phenomenon, but also an essential mechanism in tumorigenesis and progression. Previous studies have provided evidence that 
hybrid cells exhibit altered properties including increased metastatic ability and enhanced resistance to apoptosis as well as an enhanced drug resistance compared with the parental tumor cells (17-19). A recent study confirmed that fusion occurs between bone marrow-derived cells and tumor cells in human cancer (20). Cell fusion was identified in cell culture and animal studies in various tumors including melanoma, intestinal tumors, and breast carcinoma $(18,20,21)$. However, it is rarely reported in $\mathrm{HCC}$ in vivo. The present dual-fluorescent xenograft HCC model offered an easy and direct method to confirm the presence of fused cells via the expression of RFP and GFP. The fused cells observed in the present study, may be derived from the fusion of the RFP and GFP parental cells, originating from the fusion of tumor cells with normal host cells. However the function of fused cells in the development of HCC requires further investigation. As demonstrated by Fig. 5D, cell, cells which was marked with the second and third arrows were also fusion cells. Further research is required to determine whether the fused cells increase the malignancy of tumors including invasion and migration in HCC.

Metastasis represents the end product of a multistep cellular process termed the invasion metastasis cascade, which involves dissemination of cancer cells to anatomically distant organ sites and their subsequent adaptation to foreign tissue microenvironments (12). The present study confirmed that the first step in metastasis of HCC is the exit of single cells from the primary site. At a cellular level, the majority of types of carcinomas may invade as cohesive multicellular units through a process termed 'collective invasion'. Alternatively, individual tumor cells may invade via two distinct pathways: The protease-, stress fiber-, and integrin-dependent 'mesenchymal invasion' pathway or the protease-, stress fiber-, and integrin-independent, Rho/ROCK-dependent 'amoeboid invasion' pathway (22). However, it is largely unknown which pathway takes place during HCC invasion, and further investigation is required.

In conclusion, a GFP/RFP dual-color tumor model is useful for visualizing early-stage tumors and screening therapeutic agents for HCC. The TME and the connectivity of tumor cells should be investigated due to the direct interactions of tumor cells with host tissue cells during the tumor remodeling process.

\section{References}

1. Ferlay J, Shin HR, Bray F, Forman D, Mathers C and Parkin DM: Estimates of worldwide burden of cancer in 2008: GLOBOCAN 2008. Int J Cancer 127: 2893-2917, 2010.

2. El-Serag HB and Rudolph KL: Hepatocellular carcinoma: Epidemiology and molecular carcinogenesis. Gastroenterology 32: 2557-2576, 2007.

3. Bosetti C, Turati F and La Vecchia C: Hepatocellular carcinoma epidemiology. Best Pract Res Clin Gastroenterol 28 753-770, 2014.
4. Wu L, Tang ZY and Li Y: Experimental models of hepatocellular carcinoma: Developments and evolution. J Cancer Res Clin Oncol 135: 969-981, 2009.

5. Yang M, Li L, Jiang P, Moossa AR, Penman S and Hoffman RM: Dual-color fluorescence imaging distinguishes tumor cells from induced host angiogenic vessels and stromal cells. Proc Natl Acad Sci USA 100: 14259-14262, 2003.

6. Hayashi K, Yamauchi K, Yamamoto N, Tsuchiya H, Tomita K, Amoh Y, Hoffman RM and Bouvet M: Dual-color imaging of angiogenesis and its inhibition in bone and soft tissue sarcoma. J Surg Res 140: 165-170, 2007.

7. Bouvet M and Hoffman RM: In vivo imaging of pancreatic cancer with fluorescent proteins in mouse models. Methods Mol Biol 872: 51-67, 2012.

8. Yamamoto N, Tsuchiya H and Hoffman RM: Tumor imaging with multicolor fluorescent protein expression. Int J Clin Oncol 16: 84-91, 2011

9. Hoffman RM: Transgenic nude mice ubiquitously expressing fluorescent proteins for color-coded imaging of the tumor microenvironment. Methods Mol Biol 1194: 353-365, 2014.

10. Dong J, Zhang Q, Huang Q, Chen H, Shen Y, Fei X, Zhang T, Diao Y, Wu Z, Qin Z, et al: Glioma stem cells involved in tumor tissue remodeling in a xenograft model. J Neurosurg 113: 249-260, 2010

11. Wang A, Dai X, Cui B, Fei X, Chen Y, Zhang J, Zhang Q, Zhao Y, Wang Z, Chen H, et al: Experimental research of host macrophage canceration induced by glioma stem progenitor cells. Mol Med Rep 11: 2435-2442, 2015.

12. Valastyan S and Weinberg RA: Tumor metastasis: Molecular insights and evolving paradigms. Cell 147: 275-292, 2011.

13. Heindryckx F, Colle I and Van Vlierberghe H: Experimental mouse models for hepatocellular carcinoma research. Int J Exp Pathol 90: 367-386, 2009.

14. Walters DM, Stokes JB, Adair SJ, Stelow EB, Borgman CA, Lowrey BT, Xin W, Blais EM, Lee JK, Papin JA, et al: Clinical, molecular and genetic validation of a murine orthotopic xenograft model of pancreatic adenocarcinoma using fresh human specimens. PLoS One 8: e77065, 2013.

15. Zhao GJ, Xu LX, Chu ES, Zhang N, Shen JY, Damirin A and Li XX: Establishment of an orthotopic transplantation tumor model of hepatocellular carcinoma in mice. World $\mathrm{J}$ Gastroenterol 18: 7087-7092, 2012.

16. Abou-Elkacem L, Gremse F, Barth S, Hoffman RM, Kiessling F and Lederle W: Comparison of $\mu \mathrm{CT}$, MRI and optical reflectance imaging for assessing the growth of GFP/RFP-expressing tumors. Anticancer Res 31: 2907-2913, 2011.

17. Berndt B, Zänker KS and Dittmar T: Cell fusion is a potent inducer of aneuploidy and drug resistance in tumor cell/ normal cell hybrids. Crit Rev Oncog 18: 97-113, 2013.

18. Dittmar T, Schwitalla S, Seidel J, Haverkampf S, Reith G, Meyer-Staeckling S, Brandt BH, Niggemann B and Zänker KS: Characterization of hybrid cells derived from spontaneous fusion events between breast epithelial cells exhibiting stem-like characteristics and breast cancer cells. Clin Exp Metastasis 28: 75-90, 2011.

19. Pawelek JM: Fusion of bone marrow-derived cells with cancer cells: Metastasis as a secondary disease in cancer. Chin J Cancer 33: 133-139, 2014.

20. Lazova R, Laberge GS, Duvall E, Spoelstra N, Klump V, Sznol M, Cooper D, Spritz RA, Chang JT and Pawelek JM: A melanoma brain metastasis with a donor-patient hybrid genome following bone marrow transplantation: First evidence for fusion in human cancer. PLoS One 8: e66731,2013.

21. Powell AE, Anderson EC, Davies PS, Silk AD, Pelz C, Impey S and Wong MH: Fusion between intestinal epithelial cells and macrophages in a cancer context results in nuclear reprogramming. Cancer Res 71: 1497-1505, 2011.

22. Friedl P and Wolf K: Tumour-cell invasion and migration: Diversity and escape mechanisms. Nat Rev Cancer 3: 362-374, 2003. 